\title{
TECNOLOGIA ASSISTIVA NO AUXÍLIO AO IDOSO: proposta de dispenser de comprimidos para uso doméstico
}

\section{ASSISTIVE TECHNOLOGY IN THE AID TO THE ELDERLY: proposal of a dispenser for domestic use}

\author{
Rosana Polatto ${ }^{1}$, Tecg. \\ Ana Cristina Maurício Ferreira ${ }^{2}$, MsC. \\ Wangner Barbosa da Costa ${ }^{3}$, MsC. \\ Maria Aline Lemos Silva Thobias ${ }^{4}$, MsC. \\ (1) Faculdade de Tecnologia de Bauru (FATEC) \\ e-mail: redlinebauru@hotmail.com
}

(2) Universidade Estadual Paulista (UNESP), PPGDesign-FAAC

e-mail: anacr.fatec@gmail.com

\author{
(3) Faculdade de Tecnologia de Bauru (FATEC) \\ e-mail: wangnerbc@gmail.com \\ (4) Faculdade de Tecnologia de Bauru (FATEC) \\ e-mail:arthobias@uol.com.br
}

Palavras chave: Tecnologia assistiva, Envelhecimento populacional, Equipamento médico hospitalar.

\begin{abstract}
A acessibilidade a serviços, produtos ou atividades é um direito de todo ser humano, assim como a sua segurança e preservação de sua qualidade de vida. Com o avanço da idade, determinadas atividades podem se tornar complexas e inseguras, como é o caso da necessidade de se tomar medicamentos diversas vezes ao dia. Desta forma, este trabalho objetivou apresentar uma solução pautada nos conceitos da tecnologia assistiva, para o auxílio da polimedicamentação diária. Os procedimentos metodológicos envolveram o levantamento bibliográfico, a aplicação de pesquisa para identificar o uso diário de medicamentos e a ocorrência de esquecimento do uso dos mesmos e o levantamento de similares. O resultado foi o projeto de um dispositivo eletrônico programável para aplicação no controle do armazenamento, dosagem e distribuição de comprimidos. A relevância deste trabalho se deve ao fato de que a correta dosagem medicamentosa é a diferença entre remédio e veneno.
\end{abstract}

Keywords: Assistive technology, Population-Ageing, Hospital medical equipment.

Accessibility to services, products or activities is a right of every human being, as well as their safety and preservation of their quality of life. With advancing age, certain activities can become complex and insecure, such as the need to take medications several times a day. In this way, this work aimed to present a solution, based on the concepts of assistive technology for the aid of daily polymedication. The methodological procedures involved the bibliographic survey, the application of research to identify the daily use of medications and the occurrence of forgetfulness of the use of the same and the survey of similar ones. The result was the design of a programmable electronic device for application in the control of the storage, dosage and distribution of tablets. The relevance of this work is due to the fact that the correct drug dosage is the difference between medicine and venom. 


\section{$16^{\circ}$ \\ ERGODESIGN USIHC CINAHPA}

\section{Introdução}

A acessibilidade a locais, serviços ou atividades da vida diária é um direito de todo indivíduo e, conforme Scatolim et al (2015) é uma condição que depende da ergonomia, do design e outras áreas de igual importância. Através da aplicação da ergonomia e da tecnologia, é possível melhorar a qualidade de vida de pessoas que apresentam alguma necessidade especial, como é o caso de pessoas idosas.

Neste contexto, a Tecnologia Assistiva amplia habilidades funcionais deficitárias, possibilitando a realização de tarefas básicas por pessoas que se encontram, de certa forma, limitadas por circunstâncias advindas do envelhecimento, restaurando, em grande parte, sua autonomia. Diversos são os recursos e produtos desenvolvidos no intuito de promover às pessoas fragilizadas, uma vida independente, tornando-as capazes de realizar tarefas no seu cotidiano.

Estes produtos/recursos permitem que se cumpra a mais nobre das causas, o direito, concedendo oportunidades e igualdade às pessoas idosas e/ou com limitações. A possibilidade de os idosos terem esta autonomia, tende a melhorar a sua percepção da qualidade de vida.

Este trabalho trata do desenvolvimento de um dispositivo cuja função global é permitir a separação e a identificação de medicamentos (em formato de comprimidos), proporcionando segurança, autonomia e facilidade operacional às pessoas que apresentam alguma limitação física ou cognitiva na realização de tarefas, como ocorre com alguns idosos.

\subsection{Envelhecimento Populacional}

Ao envelhecer o que importa é a capacidade do idoso de desenvolver suas atividades da vida diária, sendo capaz de tomar decisões, participar e contribuir com o cenário em que está inserido. Idoso saudável não é aquele isento de alguma morbidade, mas aquele que se considera participativo em atividades na sociedade e com capacidade funcional íntegra, podendo se sentir $16^{\circ}$ Ergodesign - Congresso Internacional de Ergonomia e Usabilidade de Interfaces Humano Tecnológica: Produto, Informações Ambientes Construídos e Transporte

$16^{\circ}$ USIHC - Congresso Internacional de Ergonomia e Usabilidade de Interfaces Humano Computador

CINAHPA | 2017 - Congresso Internacional de Ambientes Hipermídia para Aprendizagem.

habilitado a fazer tarefas no seu cotidiano (FERREIRA, 2012).

Em 2011, no Brasil, a população idosa era de 20,5 milhões, o equivalente a $10,8 \%$. Projeções indicam que, em 2020, a população idosa será de 30,9 milhões, representando $14 \%$ da população total (KÜCHEMANN, 2012).

Os censos populacionais entre 2000 e 2012, mostram um índice de crescimento da população brasileira de $12,3 \%$, aproximadamente 21 milhões de pessoas, de um total contabilizado no ano de 2012 de aproximadamente 191 milhões de habitantes. A proporção de idosos na população neste mesmo período passou de 5,9\% para 7,4\%, (BRASIL, 2012). A Tabela 1 mostra o crescimento da população idosa, em relação a outras faixas etárias.

\begin{tabular}{ccc|c}
\hline & \multicolumn{3}{c}{ FAIXA ETÁRIA } \\
\cline { 2 - 4 } PERÍODO & ATÉ 14 & $\mathbf{1 5}$ A 64 & 65 ANOS \\
& ANOS & ANOS & OU MAIS \\
& $(\boldsymbol{\%})$ & $(\boldsymbol{\%})$ & $(\boldsymbol{\%})$ \\
\hline 1970 & 42,6 & 54,3 & 3,1 \\
1980 & 38,8 & 56,4 & 4,0 \\
1991 & 35,0 & 60,2 & 4,8 \\
2000 & 29,7 & 65,2 & 5,1 \\
2010 & 26,5 & 67,7 & 5,8 \\
$\mathbf{2 0 2 0}$ & $\mathbf{2 3 , 6}$ & $\mathbf{6 8 , 7}$ & $\mathbf{7 , 7}$ \\
\hline
\end{tabular}

Tabela 1. Crescimento populacional

Fonte: Brasil, IBGE, Censo demográfico de 2012

A preocupação com a saúde e o bem-estar dos idosos deve ter um olhar diferenciado devido a esta alteração no crescimento da faixa etária no mundo. Além de alterar o ciclo de vida e a relação com a transformação e o bem-estar destas pessoas, existe o conceito de isolamento. As pessoas estão cada vez mais afastadas do convívio social, principalmente nas grandes cidades, com o desafio de usar tecnologias cada vez mais sofisticadas e avançadas e garantir a segurança e longevidade, tanto no convívio familiar quanto no seu dia a dia.

O investimento em Tecnologias Assistivas no auxilio pré e pós-envelhecimento deveria ser constante (RAMOS, 2003).
Realização:

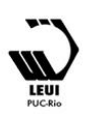


$16^{\circ}$ Ergodesign - Congresso Internacional de Ergonomia e Usabilidade de Interfaces Humano Tecnológica: Produto, Informações Ambientes Construídos e Transporte

$16^{\circ}$ USIHC - Congresso Internacional de Ergonomia e Usabilidade de Interfaces Humano Computador

CINAHPA | 2017 - Congresso Internacional de Ambientes Hipermídia para Aprendizagem.

\subsection{Tecnologia Assistiva}

A adversidade advinda do processo de envelhecimento impõe, ao idoso, o auxílio de terceiros, sejam eles cuidadores, enfermeiros ou familiares. O uso de dispositivos de Tecnologia Assistiva, empregadas no desenvolvimento de aplicações multidisciplinares e transdisciplinares na ciência, arte, medicina, design, sistemas conexionistas, cognição e neurociência, auxiliam ou mesmo permitem a realização das atividades da vida diária, proporcionando segurança e melhora na qualidade de vida (BERSCH, 2008).

Percebe-se que a evolução tecnológica muda o curso da vida de pessoas dependentes, conseguindo tornar a vida mais fácil e menos pesada com o envelhecimento, utilizando ferramentas desenvolvidas para favorecer e simplificar as atividades do cotidiano, como os óculos, canetas, computadores, controle remoto, automóveis, telefones celulares, relógio, enfim, uma interminável lista de recursos, que já estão assimilados a nossa rotina. Entretanto, é possível perceber que a aplicação de Tecnologia Assistiva requer mudanças e investimentos políticos e sociais, valores em nossa sociedade num senso geral, assim os serviços e recursos tornar-se-ão instrumentos que facilitam o desempenho em funções pretendidas. A Tecnologia Assistiva traz o entendimento de como um auxílio, promoverá a ampliação e habilidade funcional deficitária ou possibilitará a realização das funções básicas de pessoas que se encontram impedidas por questões circunstanciais do envelhecimento. As considerações até aqui apresentadas indicam que os recursos de Tecnologia Assistiva tem sido alvo de pesquisa e pesquisadores interessados em mudar os conceitos e aplicações no estudo do desenvolvimento de equipamentos de auxílio às funções físicas (BERSCH, 2008).

\subsection{Uso de Medicamentos}

Sobre a importância do uso correto de medicamentos entre pessoas idosas e com limitações, estudos deste tipo têm como finalidade desenvolver tecnologia e serviços que contribuam para promover vida independente e inclusão. $\mathrm{O}$ número de pessoas que fazem uso diário de vários medicamentos é um indicador de preocupação na hora da prescrição e da assistência médicosanitária, embora a exposição aos múltiplos fármacos não seja sinônimo de prescrição imprópria. A preocupação com a distribuição e dosagem dos fármacos depende da atuação no campo da prescrição e no campo da investigação científica, associada ao investimento em tecnologia de controle (LÚCIO, 2013).

Rozenfeld (2003) cita que a grande maioria da população idosa consome, ao menos, um medicamento por dia, sendo que cerca de um terço deles desta população consome cinco ou mais simultaneamente. A média de fármacos utilizados diariamente por idosos varia entre dois e cinco.

Flores e Mengue (2005), na realização de uma pesquisa, feita no Rio Grande do Sul, cujo objetivo era descrever o uso de medicamentos por idosos, relataram que de uma amostra de 215 idosos, de ambos os gêneros, a prevalência do uso de medicação diária foi de $91 \%$, constatando o padrão elevado de uso de medicamentos entre pessoas de faixa etária igual ou superior a 60 anos.

Neves et al (2013) em estudo realizado com 400 sujeitos idosos de ambos os gêneros da cidade de Recife/PE, que teve por objetivo a análise do uso de medicamentos entre idosos e fatores associados, mostrou que a prevalência de uso de medicamentos foi de $85,5 \%$, corroborando a existência da alta proporção de uso de medicamentos entre esta população. Deste percentual, 98,2\% dos usuários idosos de medicamentos o fazem por prescrição médica.

\section{Procedimentos metodológicos}

Os procedimentos metodológicos tiveram início em uma revisão de literatura narrativa que, conforme Rother (2007) busca publicações amplas sob o ponto de vista teórico. A partir das informações levantadas nas publicações, sua análise e classificação, a estrutura lógica sobre os tópicos que envolvem o tema apresentado segue um critério de inferência, com base no crescimento da população idosa, no uso de medicamento e sua

Realização:




\section{$16^{\circ}$ \\ ERGODESIGN USIHC CINAHPA}

importância para a vida do idoso.

Após, foi aplicada uma pesquisa junto aos idosos para verificar, além da faixa etária do sujeito participante, duas questões fechadas: se fazem uso regularmente de medicamentos e se ocorre o esquecimento ou falha no uso.

Para efeito de comparação de produtos que apresentam a mesma função global (armazenamento e distribuição de medicamentos), fez-se um levantamento de produtos similares, que serviu como base comprobatória da real necessidade de investimentos e melhorias no equipamento proposto neste projeto, a fim de propiciar mais segurança no uso de medicamentos.

No desenvolvimento foi desenvolvido um projeto conceitual das funções operacionais do dispositivo eletrônico proposto. A partir do projeto conceitual, foi elaborado o projeto elétrico do sistema, desenvolvido no CADi-Simu, demostrando o sistema de entrada, fonte de alimentação, baterias e chaveamento. As simulações foram feitas para melhor compreensão do funcionamento do projeto, sendo utilizada a programação Ladder.

\section{Resultados}

\subsection{Pesquisa de campo aplicada}

Após a abordagem do indivíduo idoso foi aplicado o termo de consentimento livre e esclarecido (CNS, 1996) e realizada uma leitura prévia da pesquisa, com esclarecimentos quanto ao motivo de sua realização. A pesquisa foi realizada com 20 sujeitos idosos, de ambos os gêneros, na faixa etária de 60 a 75 anos. O propósito da aplicação da pesquisa foi identificar o uso diário de medicamentos e a ocorrência de esquecimento do uso dos mesmos.

Foi verificado nesta pesquisa que fazem uso de medicações diariamente: i) $90 \%$ de usuários na faixa etária de 60 a 64 anos; ii) 95\% de usuários entre 65 a 69 anos e; iii) $100 \%$ dos usuários acima de 70 anos. Sobre o esquecimento, $100 \%$ dos idosos acima de 65 anos declararam esquecer de fazer o uso do medicamento regularmente. $16^{\circ}$ Ergodesign - Congresso Internacional de Ergonomia e Usabilidade de Interfaces Humano Tecnológica: Produto, Informações Ambientes Construídos e Transporte

$16^{\circ}$ USIHC - Congresso Internacional de Ergonomia e Usabilidade de Interfaces Humano Computador

CINAHPA | 2017 - Congresso Internacional de Ambientes Hipermídia para Aprendizagem.

\subsection{Levantamento de produtos similares}

O objetivo do levantamento de análise de similares, foi pesquisar por dispositivos eletrônicos que desempenham a função de armazenamento e distribuição de medicamentos. O dispositivo analisado foi o Automatic Pill Dispenser With Alarm (Figura 1).

O dispositivo tem forma cilíndrica com prato não removível, possuindo um tabuleiro que é dividido radialmente em 28 compartimentos, onde são colocados os comprimidos que serão ministrados sequencialmente. O dispositivo é configurado com os horários das ministrações.

Dois aspectos importantes foram observados: i) o tabuleiro não é removível, o que dificulta a sua higienização e manutenção; ii) utiliza apenas o alarme sonoro para aviso do horário da administração do medicamento não contendo dispositivo dispensador da dosagem.

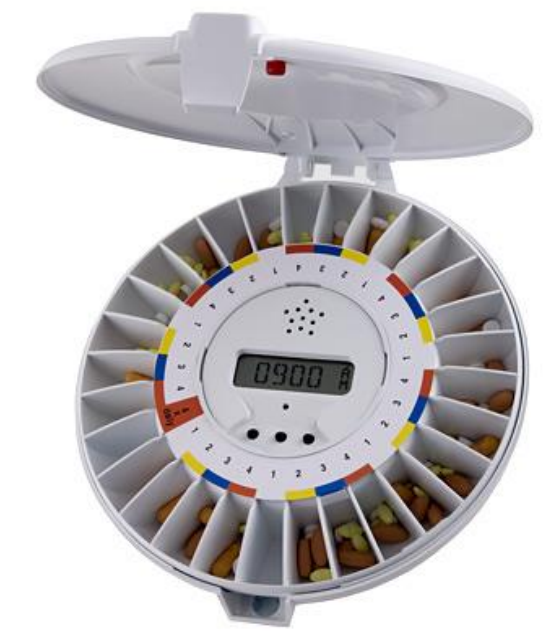

Figura 1 - Automatic Pill Dispenser With Alarm (produto similar)

Dispensadores não eletrônicos, não foram considerados neste levantamento. Outros dispositivos foram encontrados, mas com divisão diária dos medicamentos e não por ministração.

\subsection{Conceito operacional do modelo proposto}

Em todo o processo de desenvolvimento da pesquisa pensou-se em um produto portátil, de baixo custo, com boa autonomia de funcionamento
Realização:

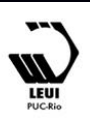




\section{$16^{\circ}$ \\ ERGODESIGN USIHC CINAHPA}

e com um sistema programável que para atender às diferentes variáveis no uso dos medicamentos.

O modelo proposto foi conceituado com as seguintes características:

a) Material do dispositivo: dispositivo fabricado em poli metil-metacrilato (acrílico), material que pode diminuir o custo do aparelho, tornando-o mais resistente, proporcionando controle de sua eficiência, devido a sua transparência e boa durabilidade. $\mathrm{O}$ compartimento onde ficarão reservados os comprimidos será fabricado em polímero maleável, evitando que no processo de girar cause desgaste ou travamento do mecanismo. Os materiais citados são de fácil higienização.

b) Forma: o formato circular deve contribuir para a distribuição dos medicamentos, girando sem encontrar barreiras, levando o medicamento até a porta de saída. Autonomia de distribuição de até 14 administrações. A colocação do medicamento é realizada seguindo uma ordem cronológica condizente com os horários e dosagens programáveis;

c) Sistema de distribuição: em bandejas giratórias programáveis e com etiquetas (que compõem o kit do equipamento) indicativas do horário em que o comprimido deve ser ministrado. As etiquetas devem fazer parte do conjunto do equipamento.

d) Compartimento de saída: somente a dosagem programada fica disponível no compartimento de saída. Neste compartimento de saída terá um sensor (sensor de movimento ou de presença infravermelho) que acionará o alarme e o sinal luminoso, permitindo que o usuário seja alertado do momento de se tomar a medicação. Ao se retirar o medicamento, tanto o sinal luminoso quanto o alarme se desligarão.

e) Reativação do sistema: assim que o medicamento for retirado, um sensor colocado no final do processo do dispensador, desligará o sistema sonoro e luminoso, e o processo de contagem será reiniciado até que outro $16^{\circ}$ Ergodesign - Congresso Internacional de Ergonomia e Usabilidade de Interfaces Humano Tecnológica: Produto, Informações Ambientes Construídos e Transporte

$16^{\circ}$ USIHC - Congresso Internacional de Ergonomia e Usabilidade de Interfaces Humano Computador

CINAHPA | 2017 - Congresso Internacional de Ambientes Hipermídia para Aprendizagem. medicamento seja dispensado;

O funcionamento deve ocorrer conforme as etapas dispostas no Quadro 1.

Quadro 1 - Etapas funcionais do dispositivo

\begin{tabular}{|c|c|}
\hline ETAPAS & DESCRIÇÃO DAS ETAPAS \\
\hline 1 & Configurar os horários através da interface; \\
\hline 2 & Ligar o dispositivo; \\
\hline 3 & $\begin{array}{l}\text { Iniciar a rotina de programa interna ao micro } \\
\text { controlador que realiza todo controle do } \\
\text { sistema; }\end{array}$ \\
\hline 4 & $\begin{array}{l}\text { Verificar o horário proveniente do circuito } \\
\text { integrado e comparar com os horários } \\
\text { programados para a liberação dos } \\
\text { medicamentos; }\end{array}$ \\
\hline 5 & $\begin{array}{l}\text { Quando o horário atual coincidir com o } \\
\text { horário de dispensa, executar a função de } \\
\text { passo que o motor deve dar para ativar a } \\
\text { angulação necessária e girar até o } \\
\text { compartimento correspondente àquele horário; }\end{array}$ \\
\hline 6 & $\begin{array}{l}\text { Após o motor girar e parar na posição que se } \\
\text { encontra o compartimento com o comprimido } \\
\text { a ser liberado, executar a parte do programa } \\
\text { onde o controle aciona o atuador, fazendo } \\
\text { abrir e liberando assim o comprimido; }\end{array}$ \\
\hline 7 & $\begin{array}{l}\text { Após } 2 \text { segundos fecha-se o atuador e o disco } \\
\text { inicia uma rotação em sentido anti-horário até } \\
\text { que o sensor indutivo detecte a carga metálica } \\
\text { do disco fazendo-o parar em sua posição de } \\
\text { referência; }\end{array}$ \\
\hline 8 & $\begin{array}{l}\text { Uma rotina interna do programa realiza a } \\
\text { execução de um cálculo para indicar qual o } \\
\text { próximo compartimento daquele tipo de } \\
\text { comprimido que deverá ser posicionado a } \\
\text { seguir para a dispensa; }\end{array}$ \\
\hline 9 & $\begin{array}{l}\text { Com a liberação do comprimido é acionado o } \\
\text { sinalizador audiovisual para alertar o paciente } \\
\text { que há um comprimido a ser ingerido; }\end{array}$ \\
\hline 10 & $\begin{array}{l}\text { Esse sinal ficará acionado por } 5 \text { minutos com } \\
\text { intermitência de acionamento de } 3 \text { segundos, } \\
\text { até que o recipiente contendo o medicamento } \\
\text { seja retirado. Se dentro do intervalo de } 5 \\
\text { minutos o recipiente for retirado o programa } \\
\text { envia o sinal de } 0 \text { volts e segue sua execução } \\
\text { normal esperando o próximo horário de } \\
\text { liberação. Em caso contrário ele executa a } \\
\text { rotina de segurança, travamento do sistema e } \\
\text { configuração e envio de SMS, a parte do } \\
\text { programa que trata das mensagens SMS é } \\
\text { responsável por configurar os comandos AP } \\
\text { do modem de SMS para enviar uma } \\
\text { mensagem de texto para o número de celular } \\
\text { cadastrado no programa; }\end{array}$ \\
\hline 11 & $\begin{array}{l}\text { Essa mensagem contém o nome do paciente e } \\
\text { o nome do medicamento, seguido de uma } \\
\text { mensagem que indica a não retirada do } \\
\text { comprimido do dispensador. }\end{array}$ \\
\hline
\end{tabular}

Realização:

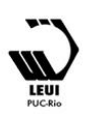




\section{$16^{\circ}$ \\ ERGODESIGN USIHC CINAHPA}

\subsection{Simulação do sistema proposto}

Após realizado o projeto elétrico, foi feita a simulação do sistema com lógica Ladder para microcontroladores PIC e AVR, que tem por características:

a) Entradas e saídas digitais;

b) Temporizadores (TON, TOF, RTO);

c) Contadores (CTU, CTD, 'contadores circulares' para usar como sequenciadores);

d) Entradas analógicas e saídas (PWM) analógicas;

e) Variáveis inteiras e instruções aritméticas;

f) Comunicação serial facilitada para conexão a PCs, LCDs e outros dispositivos;

g) Registradores de deslocamento, tabelas look-up;

h) Variáveis em EEPROM (que mantêm os dados quando falta energia elétrica);

i) Simulador, para testar seu programa antes de gerar o código PIC ou AVR;

j) É um software livre.

As telas das simulações realizadas estão apresentadas nas Figuras 2 a 7.

A Figura 2 apresenta o painel frontal do simulador Ladder, indicando o início do ciclo de tomadas. Os botões em vermelho da direita para esquerda representam o botão de ligar o equipamento (1), o botão de reset (5). Nesta etapa os comprimidos já estão no compartimento e o equipamento está pronto para escolha do ciclo de $6 \mathrm{~h}, 8 \mathrm{~h}$ ou $12 \mathrm{~h}$.

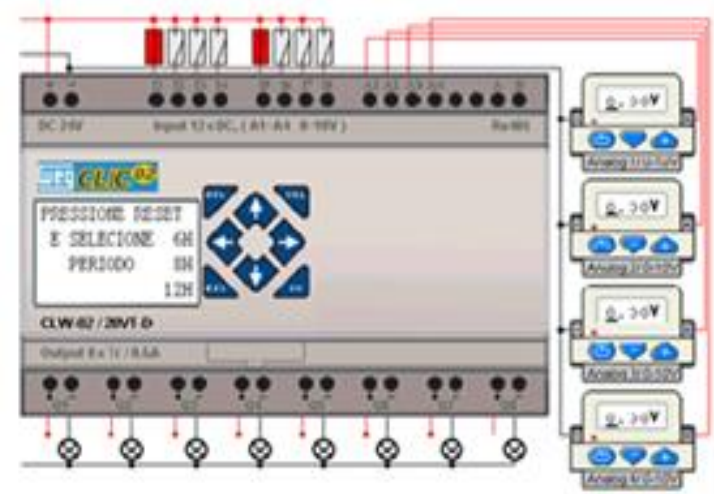

Figura 2 - Painel frontal: início do ciclo

Observa-se que enquanto não for selecionado o ciclo de administração, o equipamento não dará início à contagem e distribuição dos comprimidos. $16^{\circ}$ Ergodesign - Congresso Internacional de Ergonomia e Usabilidade de Interfaces Humano Tecnológica: Produto, Informações Ambientes Construídos e Transporte

$16^{\circ}$ USIHC - Congresso Internacional de Ergonomia e Usabilidade de Interfaces Humano Computador

CINAHPA | 2017 - Congresso Internacional de Ambientes Hipermídia para Aprendizagem.

Junto à programação CLP foi elaborado um sistema de segurança garantindo que se mais de um botão de seleção de administração for acionado, o equipamento não iniciará o ciclo de contagem, tendo o usuário de reiniciar o sistema e definir o horário de tomada novamente.

Na figura 3 é demonstrado o sistema seleção.

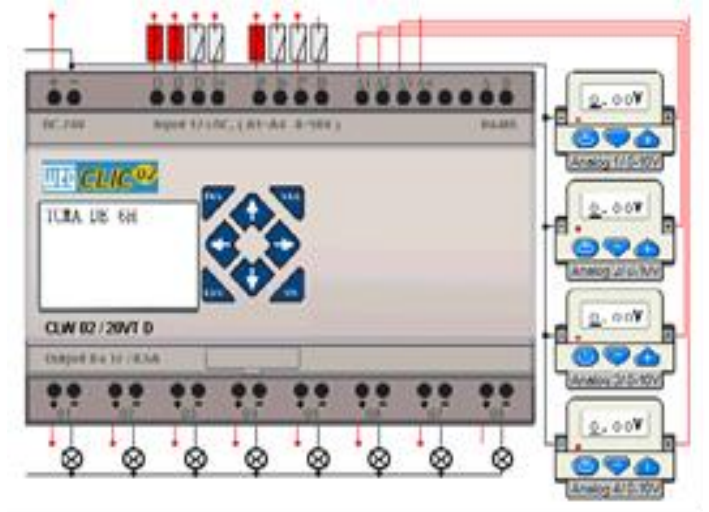

Figura 3 - Sistema de seleção

A Figura 3 demonstra o display, onde é possível perceber que o usuário já fez a seleção do ciclo e dispensa de comprimidos, selecionando o segundo botão (2) da direita para a esquerda no painel, este acionamento identificado pelo sistema como ciclo de $6 \mathrm{~h}$.

A partir deste estágio o equipamento funciona automaticamente, e o motor será acionado para fazer o giro até o compartimento de saída no ciclo selecionado, garantindo assim a entrega dos comprimidos no horário de dosagem correta. No modo $6 \mathrm{~h}$ foi simulado uma autonomia de uma semana de tomada, garantindo mais comodidade à pessoa que cuida do idoso. Este modo garante que o usuário receberá seu medicamento corretamente por 7 dias.

A diferença entre um ciclo e outro é sua autonomia de dias em que o equipamento não precisará ser abastecido com os comprimidos. O modo de $8 \mathrm{~h}$ garante uma maior autonomia, o equipamento entrega os medicamentos por 9 dias, em proporção aos outros o ciclo de $12 \mathrm{~h}$ é o mais longo e garante ao usuário uma autonomia de 14 dias de administração segura.

Como pode ser visto na Figura 4, o sistema confere
Realização:
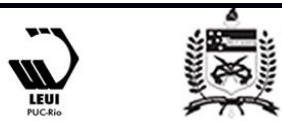


\section{$16^{\circ}$ \\ ERGODESIGN USIHC CINAHPA}

a data e hora da entrega dos medicamentos.

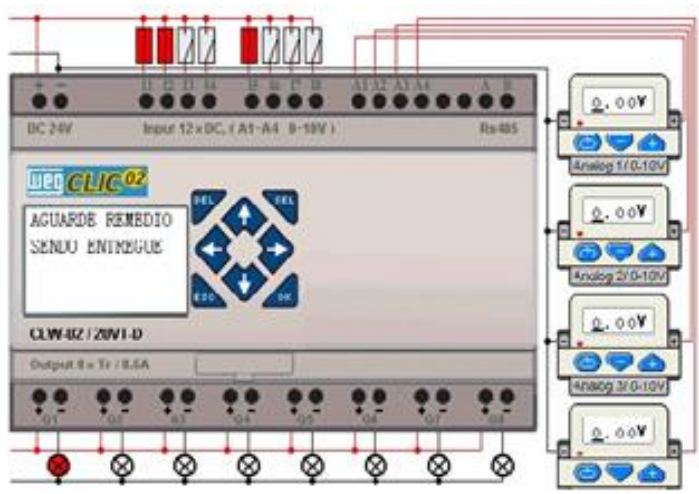

Figura 4 - Preparação para a entrega do medicamento

Na Figura 4 pode-se notar que o motor completou o giro e respondeu ao ciclo selecionado, com a identificação na parte inferior do acendimento do símbolo motor, possibilitando a visualização do funcionamento desta fase, o equipamento confere o horário e inicia a fase da dispensa dos comprimidos.

A Figura 5 mostra a entrega dos comprimidos. Nesta etapa da simulação o segundo motor é acionado como demonstrado na parte inferior do painel no símbolo motor o segundo da direita para esquerda aceso em vermelho. Nesta representação é demonstrado que o medicamento foi projetado no compartimento de espera e o sistema passa a acionar os componentes do alarme visual e sonoro.

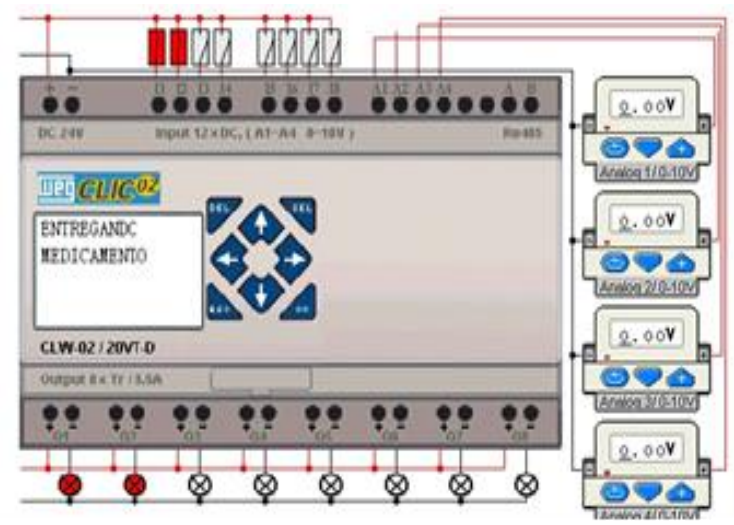

Figura 5 - Entrega dos comprimidos

Na Figura 6 é demonstrada a simulação de aviso no painel. $16^{\circ}$ Ergodesign - Congresso Internacional de Ergonomia e Usabilidade de Interfaces Humano Tecnológica: Produto, Informações Ambientes Construídos e Transporte

$16^{\circ}$ USIHC - Congresso Internacional de Ergonomia e Usabilidade de Interfaces Humano Computador

CINAHPA | 2017 - Congresso Internacional de Ambientes Hipermídia para Aprendizagem.

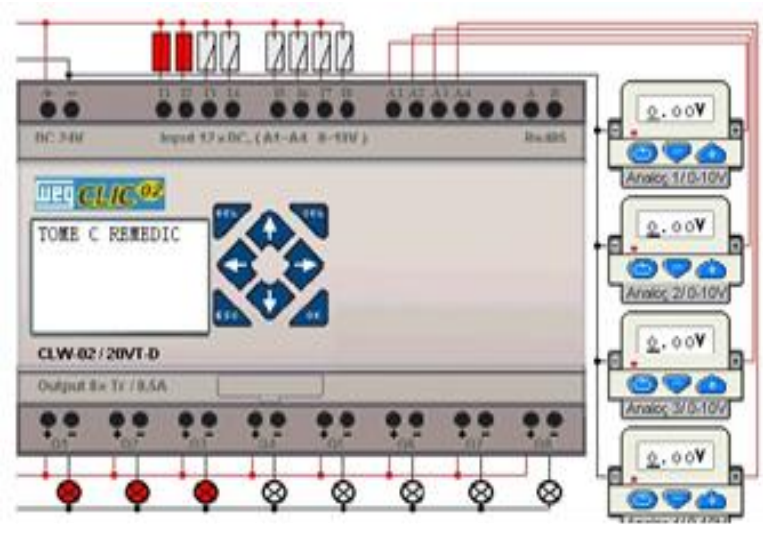

Figura 6 - Aviso sonoro e escrito

Na Figura 6 pode-se perceber o acionamento do terceiro motor identificado na terceira figura na parte inferior do painel identificada pela simbologia do motor aceso em vermelho. Nesta fase o equipamento avisa ao usuário através de sinal sonoro e escrito no painel que o medicamento está pronto e entregue e deve ser tomado.

A Figura 7 apresenta o final de ciclo e segurança.

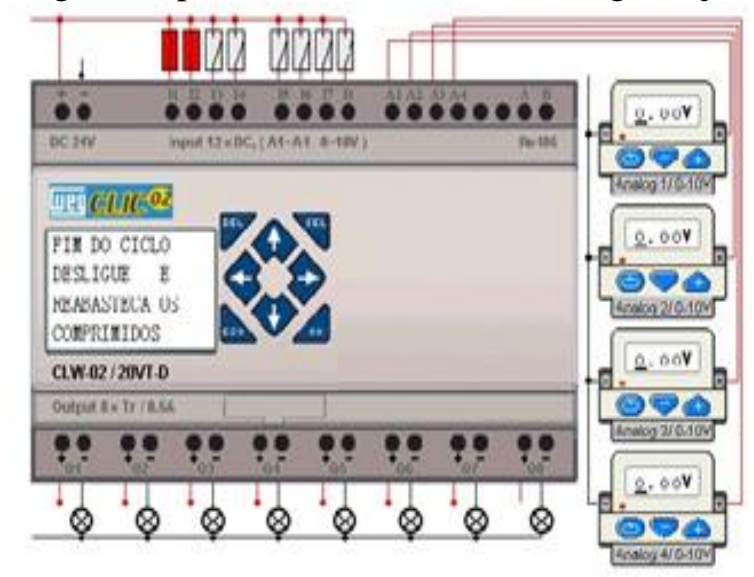

Figura 7 - Final do ciclo de entrega

Na Figura 7 o sistema demonstra o encerramento do ciclo de tomadas que teve uma autonomia de 7 dias de entrega dos comprimidos, ao finalizar o processo a programação de segurança trava o sistema, não permitindo que o usuário acione novo ciclo sem abastecer o reservatório de comprimidos e seguir o procedimento identificado no painel frontal. Com fim de ciclo, o equipamento deve ser reiniciado e reabastecido com novas doses no compartimento, e em seguida, selecionando um novo período de tomada. 


\section{$16^{\circ}$ \\ ERGODESIGN USIHC CINAHPA}

\section{Conclusão}

A metodologia aplicada, foi bastante satisfatória, proporcionando o resultado esperado com as simulações no programa Ladder. A pesquisa de campo ajudou a conhecer o percentual de idosos que fazem uso de medicação diariamente e o quanto é habitual o esquecimento da administração nos horários e dias corretos. A satisfação em combinar tecnologia com a necessidade de pessoas idosas também foi motivadora para esta pesquisa que, de certa forma, contribui para o desenvolvimento de projetos na área da tecnologia assistiva e para uma maior compreensão dos elementos que envolvem o objeto do projeto em questão. $\mathrm{O}$ fato deste dispositivo proposto ser voltado ao público idoso, visa a promoção da segurança e da qualidade de vida deste público. A não limitação formal da ação projetual, neste caso, teve o intuito de apresentar uma solução completa, embasada nos conceitos do design, da tecnologia assistiva e do conhecimento tecnológico atual.

\section{Referências}

BERSCH, R. Introdução à tecnologia assistiva. Porto Alegre: CEDI, 2008.

BRASIL. IBGE. (Org.). Instituto Brasileiro de Geografia e Estatística. 2012. Disponível em: <http://biblioteca.ibge.gov.br/visualizacao/periodic os/20/aeb_2012.pdf>. Acesso em: 08 dez. 2015.

\section{BRASIL. MINISTÉRIO DA SAÚDE. Estatuto do} idoso. 2007. Disponível em:

<http://conselho.saude.gov.br/biblioteca/livros/esta tuto_idoso2edicao.pdf $>$. Acesso em: 16 jun. 2016.

CNS. CONSELHO NACIONAL DE SAÚDE. Resolução No 196, de 10 de outubro de 1996, 1996.

FERREIRA, O. G. L. et al. Envelhecimento ativo e sua relação com a independência funcional. Texto Contexto Enferm, v. 21, n. 3, p. 513-8, 2012.

FLORES, L. M.; MENGUE, S. S. Uso de medicamentos por idosos em região do sul do Brasil. Revista de Saúde Pública, v. 39, n. 6, p.
924-929, 2005.

KÜCHEMANN, B. A. Envelhecimento populacional, cuidado e cidadania: velhos dilemas e novos desafios. 2012. Disponível em: $<$ http://www.scielo.br/scielo.php?script=sci_arttext \&pid=S0102-69922012000100010 $>$. Acesso em: 15 abr. 2016.

\section{LADDER. Lógica Ladder para}

microcontroladores PIC and AVR. 2007.

Disponível em: <http://cq.cx/ladder-pt.html>.

Acesso em: 12 out. 2016.

LÚCIO, C. C. Embalagens de Medicamentos:

diretrizes para o desenvolvimento. 2013.

Disponível em:

<http://repositorio.unesp.br/handle/11449/110872.

Acesso em: 02 mar. 2016.

NEVES, S. J. F. et al. Epidemiologia do uso de medicamentos entre idosos em área urbana do Nordeste do Brasil. Revista de Saúde Pública, v. 47, n. 4, p. 759-768, 2013.

RAMOS, L. R. Fatores determinantes do envelhecimento saudável em idosos residentes em centro urbano: Projeto Epidoso, São Paulo. 2003. Disponível em: <http://www.equipesaudepramover.com.br/artigos/ fatores-determinantes-do-envelhecimento.pdf $>$. Acesso em: 19 mar. 2016.

ROTHER, E. T. Revisão sistemática X revisão narrativa. Acta Paulista de Enfermagem, v. 20, n. 2, p. v-vi, 2007.

ROZENFELD, S. Prevalência, fatores associados e mau uso de medicamentos entre os idosos: uma revisão. Cad. Saúde Pública, v. 19, n. 3, p. 717724, 2003.

SCATOLIM, R. S. et al. Design e tecnologia assistiva: contribuições para o desenvolvimento de cadeiras de rodas inteligentes. Blucher Design Proceedings, v. 2, n. 1, p. 461-469, 2015.

Realização:

